Acta Math. Hungar., 118 (1-2) (2008), 115-127.

DOI: $10.1007 / \mathrm{s} 10474-007-6183-6$

First published online July 11, 2007

\title{
ASYMPTOTICS OF DERIVATIVES OF ORTHOGONAL POLYNOMIALS ON THE REAL LINE
}

\author{
E. LEVIN and D. S. LUBINSKY ${ }^{1 *}$ \\ ${ }^{1}$ Department of Mathematics, Mathematics Department, The Open University of Israel, \\ P.O. Box 808, Raanana 43107, Israel \\ e-mail: elile@openu.ac.il \\ 2 School of Mathematics, Georgia Institute of Technology, Atlanta, GA 30332-0160, USA \\ e-mail: lubinsky@math.gatech.edu
}

(Received September 11, 2006; accepted January 15, 2007)

\begin{abstract}
We show that uniform asymptotics of orthogonal polynomials on the real line imply uniform asymptotics for all their derivatives. This is more technically challenging than the corresponding problem on the unit circle. We also examine asymptotics in the $L_{2}$ norm.
\end{abstract}

\section{Results}

Let $\mu$ be a finite positive Borel measure on $[-1,1]$ and let $\left\{p_{n}\right\}_{n=0}^{\infty}$ denote the corresponding orthonormal polynomials, so that

$$
\int_{-1}^{1} p_{n} p_{m} d \mu=\delta_{m n}
$$

Asymptotics for derivatives of $p_{n}$ have been established under various hypotheses [1], [2], [9], [10], [13]. Many of these results deal with orthogonal polynomials on the unit circle. Recall that corresponding to $\mu$, we may define

\footnotetext{
${ }^{*}$ Research supported by NSF grant DMS0400446 and US-Israel BSF grant 2004353.

Key words and phrases: derivatives of orthogonal polynomials, asymptotics of orthogonal polynomials, Szegö's condition.

2000 Mathematics Subject Classification: primary 42C05, 42C99, secondary 41A60.
} 
a measure $\sigma$ on the unit circle by

$$
d \sigma(\theta)=d(\mu(\cos \theta)), \quad \theta \in[-\pi, \pi] .
$$

The absolutely continuous components of the two measures are connected by

$$
\sigma^{\prime}(\theta)=\mu^{\prime}(\cos \theta)|\sin \theta| .
$$

Let $\left\{\varphi_{n}\right\}$ denote the orthonormal polynomials for $\sigma$, so that

$$
\frac{1}{2 \pi} \int_{0}^{2 \pi} \varphi_{n}\left(e^{i \theta}\right) \overline{\varphi_{m}\left(e^{i \theta}\right)} d \sigma(\theta)=\delta_{m n} .
$$

The positive leading coefficient of $\varphi_{n}$ is denoted $\kappa_{n}$. In analysing $\left\{\varphi_{n}\right\}$, their reversed cousins $\varphi_{n}^{*}$ play a useful role:

$$
\varphi_{n}^{*}(z)=z^{n} \overline{\varphi_{n}(1 / \bar{z})} .
$$

We also need the monic orthogonal polynomials

$$
\Phi_{n}(z)=\varphi_{n}(z) / \kappa_{n}=z^{n}+\cdots .
$$

In a recent paper [6], the second author proved that uniform asymptotics for $\varphi_{n}$ imply uniform asymptotics for the derivatives of $\varphi_{n}$. More precisely, the following was proved, for general measures on the unit circle, that are not necessarily linked with some orthogonal polynomials on the real line:

TheOREm 1. Let $J$ be a subinterval of $[0,2 \pi]$, and assume that

$$
\lim _{n \rightarrow \infty} \varphi_{n}^{*}\left(e^{i \theta}\right)=g(\theta),
$$

uniformly for $\theta \in J$, where $g(\theta) \neq 0$ for $\theta \in J$. Let $m \geqq 1$ and $I \subset J^{0}$ be a closed interval. Then uniformly for $z=e^{i \theta}, \theta \in I$,

$$
\lim _{n \rightarrow \infty} z^{m} \varphi_{n}^{(m)}(z) /\left(n^{m} \varphi_{n}(z)\right)=1 .
$$

The proof of this involves reworking ideas from a 1979 paper of Paul Nevai [10]. It was also proved that ratio asymptotics for $\left\{\Phi_{n}\right\}$ imply ratio asymptotics for their derivatives.

In this paper, we prove analogous results for orthogonal polynomials on the real line. However, the formulation is more complex, because of the more complicated form of the asymptotics. Assuming Szegó's condition on the real line

$$
\int_{-1}^{1} \frac{\log \mu^{\prime}(x)}{\sqrt{1-x^{2}}} d x>-\infty
$$


one can form the Szegó function

$$
D(z)=\exp \left(-\frac{1}{4 \pi} \int_{-\pi}^{\pi} \log \sigma^{\prime}(\theta) \frac{z+e^{i \theta}}{z-e^{i \theta}} d \theta\right)
$$

where $\sigma^{\prime}$ is given by (1.1). The standard Szegó asymptotic for $p_{n}$ has the form

$$
p_{n}(x)=\sqrt{\frac{2}{\pi}} \operatorname{Re}\left(z^{n} / D\left(z^{-1}\right)\right)+o(1),
$$

as $n \rightarrow \infty$. Here and throughout, $x, \theta$ and $z$ are connected by the relation

$$
x=\cos \theta ; \quad z=e^{i \theta} .
$$

The Szegó condition guarantees that (1.4) holds in an $L_{2}$ sense, but not necessarily pointwise. For pointwise or uniform asymptotics, one typically needs some smoothness on $w$, such as a local $L_{2}$ Lipschitz condition [4].

The relation (1.4) helps to explain the form of the hypothesis in the following theorem. In its formulation, and throughout the paper, we use the notation

$$
\mathcal{I}_{r} f(z)= \begin{cases}\operatorname{Re} f(z), & \text { if } r \text { is even } \\ \operatorname{Im} f(z), & \text { if } r \text { is odd. }\end{cases}
$$

We assume in the sequel that $\left\{p_{n}\right\}$ are the orthonormal polynomials corresponding to the measure $\mu$, and that $\sigma$ is the corresponding measure on the unit circle, with orthonormal polynomials $\left\{\varphi_{n}\right\}$ and monic orthogonal polynomials $\left\{\Phi_{n}\right\}$. We also let $[x]$ denote the greatest integer $\leqq x$. Thus for a positive integer $r$, we have

$$
(-1)^{[r / 2]}= \begin{cases}(-1)^{r / 2}, & \text { if } r \text { is even } \\ (-1)^{(r-1) / 2}, & \text { if } r \text { is odd. }\end{cases}
$$

We use $\mathcal{D}=\frac{d}{d \theta}$, which should not be confused with the Szegó function $D(z)$. Finally, if $I$ is a subinterval of $[-1,1]$, then $\tilde{I}=\{\theta \in[0, \pi]: \cos \theta \in I\}$ is the image of $I$ under the function arc cos, while $\hat{I}=\left\{e^{i \theta}: \theta \in \tilde{I}\right\}$ is the projection of $I$ onto the unit circle.

Theorem 2. Let $\mu$ be a positive Borel measure on $[-1,1]$. Assume that $I$ is a closed subinterval of $(-1,1)$, and uniformly for $x=\cos \theta \in I$, we have as $n \rightarrow \infty$,

$$
p_{n}(x)=\operatorname{Re}\left(z^{n} f(z)\right)+o(1),
$$


where $f$ is bounded in $\hat{I}$. Assume moreover, that

$$
\lim _{n \rightarrow \infty} \Phi_{n}(0)=0 .
$$

Let $r \geqq 1$ and $I_{1}$ be a closed subinterval of $I^{0}$. Then uniformly for $x \in I_{1}$,

$$
n^{-r}\left(1-x^{2}\right)^{\frac{r}{2}}(-1)^{\left[\frac{r}{2}\right]} p_{n}^{(r)}(x)=\mathcal{I}_{r}\left(z^{n} f(z)\right)+o(1) .
$$

Note that if $\mu^{\prime}$ is positive a.e. in $(-1,1)$, then $(1.8)$ is true [11, p. 467], so we have:

COROLlary 3. Assume the hypotheses of Theorem 2, except that instead of $(1.8)$, we assume that $\mu^{\prime}$ is positive a.e. in $(-1,1)$. Then the conclusion of Theorem 2 is true.

Thus Theorem 2 asserts that once we have uniform asymptotics for orthogonal polynomials, we also obtain uniform asymptotics for their derivatives.

We shall also study mean asymptotics of derivatives of orthogonal polynomials. As far as the authors are aware, this has not been studied in general.

THEOREM 4. Let $\mu$ be a positive absolutely continuous Borel measure on $[-1,1]$ satisfying Szegô's condition (1.2). Assume, moreover, that $\sigma$ admits the following Markov-Bernstein inequality: for $n \geqq 1$, and all trigonometric polynomials $R$ of degree $\leqq n$,

$$
\left[\int_{-\pi}^{\pi}\left|R^{\prime}\right|^{2} d \sigma\right]^{1 / 2} \leqq C n\left[\int_{-\pi}^{\pi}|R|^{2} d \sigma\right]^{1 / 2} .
$$

Let $r \geqq 1$. Let $D$ be the Szegö function defined by (1.3). Then

$$
\lim _{n \rightarrow \infty} \int_{0}^{\pi}\left|n^{-r}(-1)^{\left[\frac{r}{2}\right]}\left(\frac{d}{d \theta}\right)^{r}\left[p_{n}(\cos \theta)\right]-\sqrt{\frac{2}{\pi}} \mathcal{I}_{r}\left(z^{n} / D\left(z^{-1}\right)\right)\right|^{2} d \sigma(\theta)=0
$$

and for each compact subinterval I of $(-1,1)$,

$$
\lim _{n \rightarrow \infty} \int_{I}\left|n^{-r}\left(1-x^{2}\right)^{\frac{r}{2}}(-1)^{\left[\frac{r}{2}\right]} p_{n}^{(r)}(x)-\sqrt{\frac{2}{\pi}} \mathcal{I}_{r}\left(z^{n} / D\left(z^{-1}\right)\right)\right|^{2} d \mu(x)=0 .
$$

COROLlary 5. Under the hypotheses of Theorem 4,

$$
\lim _{n \rightarrow \infty} \int_{-1}^{1}\left|n^{-1}\left(1-x^{2}\right)^{\frac{1}{2}} p_{n}^{\prime}(x)-\sqrt{\frac{2}{\pi}} \operatorname{Im}\left(z^{n} / D\left(z^{-1}\right)\right)\right|^{2} d \mu(x)=0 .
$$


It is not clear that $(1.12)$ holds with $I=(-1,1)$ and $r \geqq 2$ without additional assumptions on $w$, such as further Markov-Bernstein or Schur inequalities. One can already observe some of the difficulties for $r=2$ :

$$
\mathcal{D}^{2}\left[p_{n}(\cos \theta)\right]-p_{n}^{\prime \prime}(\cos \theta)(\sin \theta)^{2}=-p_{n}^{\prime}(\cos \theta) \cos \theta=\cot \theta \mathcal{D}\left[p_{n}(\cos \theta)\right],
$$

and the term $\cot \theta$ becomes unbounded near the endpoints of $[0, \pi]$. For Jacobi weights, one can verify that the requisite estimates hold.

The hypothesis (1.10) holds for Jacobi weights, generalized Jacobi weights, and still more generally, the doubling weights of Mastroianni and Totik [7]. It is likely that there are Szegő weights violating (1.10), but we do not have an explicit example.

We shall also state a local version of Theorem 4 :

THEOREM 6. Let $\mu$ be a positive absolutely continuous Borel measure on $[-1,1]$ satisfying Szegô's condition (1.2). Assume that $L$ is a closed subinterval of $[-1,1]$ in which $\sigma$ admits the following Markov-Bernstein inequality: for $n \geqq 1$, and all trigonometric polynomials $R$ of degree $\leqq n$,

$$
\left[\int_{\tilde{L}}\left|R^{\prime}\right|^{2} d \sigma\right]^{1 / 2} \leqq C n\left[\int_{-\pi}^{\pi}|R|^{2} d \sigma\right]^{1 / 2} .
$$

Then the conclusion (1.11) holds if $(0, \pi)$ is replaced by $\tilde{L}$, while (1.12) holds for any closed subinterval $I$ of $L^{0}$.

In particular, if $\sigma^{\prime}$ is bounded above and below by positive constants in some closed interval $L_{1}$, then the hypothesis (1.14) of Theorem 6 is satisfied with $L$ taken as any compact subinterval of the interior of $L_{1}$. In the sequel $C, C_{1}, C_{2}, \ldots$ denote constants independent of $n, x, \theta$. The same symbol does not necessarily denote the same constant in different occurrences. We shall write $C=C(\alpha)$ or $C \neq C(\alpha)$ to denote dependence on, or independence of, $\alpha$, respectively.

\section{Proof of Theorem 2}

In the proof of Theorem 2, we need the polynomials $\left\{q_{n}\right\}$, orthonormal with respect to the weight $\left(1-x^{2}\right) w(x)$ :

$$
\int_{-1}^{1} q_{n}(x) q_{m}(x)\left(1-x^{2}\right) w(x) d x=\delta_{m n} .
$$

We also set

$$
\beta_{m}=\frac{1}{\sqrt{2 \pi}}\left(1+\Phi_{m}(0)\right)^{-1 / 2} ; \quad \lambda_{m}=\frac{1}{\sqrt{2 \pi}}\left(1-\Phi_{m}(0)\right)^{-1 / 2} .
$$


The most important idea is to represent $\varphi_{n}^{*}$ in terms of $p_{n}$ alone. The ideas to do this are contained in a paper of Máté, Nevai and Totik [8, p. $262 \mathrm{ff}$.], although the identity in (b) below is not stated in the form there.

LEMma. (a)

$$
\begin{gathered}
p_{n}(x)=\beta_{2 n}\left\{z^{-n} \varphi_{2 n}(z)+z^{n} \varphi_{2 n}\left(\frac{1}{z}\right)\right\} \\
q_{n}(x)=2 \lambda_{2 n+2} \frac{z^{-n-1} \varphi_{2 n+2}(z)-z^{n+1} \varphi_{2 n+2}\left(\frac{1}{z}\right)}{z-z^{-1}} .
\end{gathered}
$$

(b)

$$
2 z^{n} \varphi_{2 n}^{*}\left(\frac{1}{z}\right)=\frac{i}{\sqrt{1-x^{2}}}\left[\frac{z^{-1} p_{n}(x)}{\beta_{2 n}}-\frac{p_{n+1}(x)}{\beta_{2 n+2}}+\operatorname{Re}\left(\eta_{n}(z)\right)\right],
$$

where

$$
\eta_{n}(z)=2 z^{-n-1} \varphi_{2 n}(z)\left[\frac{\varphi_{2 n+2}(z)}{\varphi_{2 n}(z)}-z^{2}\right] .
$$

Proof. (a) See [12, p. 294].

(b) Since $z-z^{-1}=2 i \sqrt{1-x^{2}}$, we can rewrite the identities of (a) as

$$
\begin{gathered}
\frac{p_{n}(x)}{\beta_{2 n}}=z^{-n} \varphi_{2 n}(z)+z^{n} \varphi_{2 n}\left(\frac{1}{z}\right) ; \\
i \frac{q_{n-1}(x)}{\lambda_{2 n}} \sqrt{1-x^{2}}=z^{-n} \varphi_{2 n}(z)-z^{n} \varphi_{2 n}\left(\frac{1}{z}\right) .
\end{gathered}
$$

We add these to obtain

$$
\frac{p_{n}(x)}{\beta_{2 n}}+i \frac{q_{n-1}(x)}{\lambda_{2 n}} \sqrt{1-x^{2}}=2 z^{-n} \varphi_{2 n}(z)
$$

and hence

$$
\frac{p_{n+1}(x)}{\beta_{2 n+2}}+i \frac{q_{n}(x)}{\lambda_{2 n+2}} \sqrt{1-x^{2}}=2 z^{-n-1} \varphi_{2 n+2}(z) .
$$

We multiply the second last equation by $z$ and subtract it from the last equation, to obtain

$$
\left[\frac{p_{n+1}(x)}{\beta_{2 n+2}}-z \frac{p_{n}(x)}{\beta_{2 n}}\right]+i \sqrt{1-x^{2}}\left[\frac{q_{n}(x)}{\lambda_{2 n+2}}-z \frac{q_{n-1}(x)}{\lambda_{2 n}}\right]
$$




$$
=2 z^{-n-1} \varphi_{2 n+2}(z)-2 z^{-n+1} \varphi_{2 n}(z)=\eta_{n}(z) .
$$

Now take real parts:

$$
\left[\frac{p_{n+1}(x)}{\beta_{2 n+2}}-x \frac{p_{n}(x)}{\beta_{2 n}}\right]+\left(1-x^{2}\right) \frac{q_{n-1}(x)}{\lambda_{2 n}}=\operatorname{Re}\left(\eta_{n}(z)\right)
$$

and hence

$$
\left(1-x^{2}\right) \frac{q_{n-1}(x)}{\lambda_{2 n}}=\operatorname{Re}\left(\eta_{n}(z)\right)-\left[\frac{p_{n+1}(x)}{\beta_{2 n+2}}-x \frac{p_{n}(x)}{\beta_{2 n}}\right] .
$$

Then (2.5) gives

$$
\begin{aligned}
2 z^{-n} \varphi_{2 n}(z) & =\frac{p_{n}(x)}{\beta_{2 n}}+\frac{i}{\sqrt{1-x^{2}}}\left[\operatorname{Re}\left(\eta_{n}(z)\right)-\left[\frac{p_{n+1}(x)}{\beta_{2 n+2}}-x \frac{p_{n}(x)}{\beta_{2 n}}\right]\right] \\
= & \frac{i}{\sqrt{1-x^{2}}}\left[\frac{p_{n}(x)}{\beta_{2 n}} z^{-1}-\frac{p_{n+1}(x)}{\beta_{2 n+2}}+\operatorname{Re}\left(\eta_{n}(z)\right)\right] .
\end{aligned}
$$

As $\varphi_{2 n}$ has real coefficients, we see that

$$
z^{-n} \varphi_{2 n}(z)=z^{n} \varphi_{2 n}^{*}\left(\frac{1}{z}\right)
$$

and then the result follows (cf. [4], p. 189, Lemma 1.3).

Proof of Theorem 2. We have by our hypothesis (1.8),

$$
\lim _{n \rightarrow \infty} \beta_{n}=\frac{1}{\sqrt{2 \pi}}=\lim _{n \rightarrow \infty} \lambda_{n}
$$

By standard results [11, pp. 91-92], (1.8) also ensures that

$$
\lim _{n \rightarrow \infty} \frac{\varphi_{n+1}(z)}{z \varphi_{n}(z)}=1
$$

uniformly in $\{z:|z| \geqq 1\}$, and hence uniformly in the same region,

$$
\lim _{n \rightarrow \infty} \frac{\varphi_{2 n+2}(z)}{z^{2} \varphi_{2 n}(z)}=1
$$

Then uniformly for $z \in \hat{I}, \eta_{n}(z)=o\left(\left|\varphi_{2 n}(z)\right|\right)$. 
Next, the boundedness of $f$, and our assumed asymptotic (1.7) for $\left\{p_{n}\right\}$ give for $n \geqq 1$,

$$
\left|p_{n}(x)\right| \leqq C|f(z)|+C \leqq C_{1} .
$$

Then (2.6) implies also that

$$
\left|\varphi_{2 n}(z)\right| \leqq C\left[1+\left|\eta_{n}(z)\right|\right] \leqq C\left[1+o\left(\left|\varphi_{2 n}(z)\right|\right)\right] .
$$

Then $\left\{\varphi_{2 n}\right\}_{n}$ must be uniformly bounded in $\hat{I}$, and so $\lim _{n \rightarrow \infty} \eta_{n}(z)=0$, uniformly in $\hat{I}$. From (2.3), we now deduce that uniformly in $\hat{I}$,

$$
\begin{gathered}
2 z^{n} \varphi_{2 n}^{*}\left(\frac{1}{z}\right)=\frac{i}{\sqrt{1-x^{2}}}\left[\frac{z^{-1} p_{n}(x)}{\beta_{2 n}}-\frac{p_{n+1}(x)}{\beta_{2 n+2}}+o(1)\right] \\
=\sqrt{2 \pi} \frac{i}{\sqrt{1-x^{2}}}\left[p_{n}(x) z^{-1}-p_{n+1}(x)\right]+o(1) .
\end{gathered}
$$

Now write, for a given $x$, and $n, z^{n} f(z)=a+i b$. Then

$$
\operatorname{Re}\left(z^{n+1} f(z)\right)=\operatorname{Re}(z(a+i b))=a x-b \sqrt{1-x^{2}},
$$

Substituting our assumed asymptotics for $p_{n}$ into (2.7), and using these last observations,

$$
\begin{gathered}
\sqrt{\frac{2}{\pi}}\left(1-x^{2}\right) z^{n} \varphi_{2 n}^{*}\left(\frac{1}{z}\right)=i\left[a z^{-1}-a x+b \sqrt{1-x^{2}}\right]+o(1) \\
=\sqrt{1-x^{2}}[a+i b]+o(1)=\sqrt{1-x^{2}} z^{n} f(z)+o(1) .
\end{gathered}
$$

Thus uniformly for $z \in \hat{I}$,

$$
\varphi_{2 n}^{*}\left(\frac{1}{z}\right)=\sqrt{\frac{\pi}{2}} f(z)+o(1)
$$

and hence uniformly for $z$ such that $\bar{z} \in \hat{I}$,

$$
\varphi_{2 n}^{*}(z)=\sqrt{\frac{\pi}{2}} f\left(\frac{1}{z}\right)+o(1) .
$$

From this, we deduce that uniformly for $z$ such that $\bar{z} \in \hat{I}$,

$$
\varphi_{2 n}^{*}(z)-\varphi_{2[\sqrt{n}]}^{*}(z)=o(1) .
$$


Since $\varphi_{n}$ has real coefficients, this also implies that uniformly for $z \in \hat{I}$,

$$
\varphi_{2 n}^{*}(z)-\varphi_{2[\sqrt{n}]}^{*}(z)=o(1) .
$$

Now let $I_{1}$ be a closed subinterval of $I^{0}$. Recall our notation $\mathcal{D}=\frac{d}{d \theta}$. Local Markov-Bernstein inequalities [3, pp. 242-243] give in $\hat{I}_{1}$,

$$
\left\|\mathcal{D}^{\ell}\left(\varphi_{2 n}^{*}-\varphi_{2[\sqrt{n}]}^{*}\right)\right\|_{L_{\infty}\left(\hat{I}_{1}\right)}=o\left(n^{\ell}\right)
$$

and also

$$
\left\|\mathcal{D}^{\ell} \varphi_{2[\sqrt{n}]}^{*}\right\|_{L_{\infty}\left(\hat{I}_{1}\right)}=O(\sqrt{n})^{\ell} .
$$

Combining these gives

$$
\left\|\mathcal{D}^{\ell} \varphi_{2 n}^{*}\right\|_{L_{\infty}\left(\hat{I}_{1}\right)}=o\left(n^{\ell}\right), \quad \ell \geqq 1 .
$$

Differentiating the relation

$$
p_{n}(\cos \theta)=2 \beta_{2 n} \operatorname{Re}\left[e^{-i n \theta} \varphi_{2 n}^{*}\left(e^{i \theta}\right)\right]
$$

which follows from (2.1), and using Leibniz's formula, we obtain, uniformly for $\theta \in \tilde{I}_{1}$,

$$
\begin{gathered}
\mathcal{D}^{r}\left[p_{n}(\cos \theta)\right]=2 \beta_{2 n} \sum_{j=0}^{r}\left(\begin{array}{l}
r \\
j
\end{array}\right) \operatorname{Re}\left[(-i n)^{r-j} e^{-i n \theta} \mathcal{D}^{j}\left[\varphi_{2 n}^{*}\left(e^{i \theta}\right)\right]\right] \\
=2 \beta_{2 n} \operatorname{Re}\left[(-i n)^{r} e^{-i n \theta} \varphi_{2 n}^{*}\left(e^{i \theta}\right)\right]+o\left(n^{r}\right) .
\end{gathered}
$$

In particular then,

$$
\sup _{\theta \in \tilde{I}_{1}}\left|\mathcal{D}^{r}\left[p_{n}(\cos \theta)\right]\right| \leqq C n^{r}
$$

Next, Faa di Bruno's formula for derivatives of a composition of functions [ 5 , p. 19], gives

$$
\mathcal{D}^{r}\left[p_{n}(\cos \theta)\right]
$$

$$
=\sum \frac{r !}{j_{1} ! j_{2} ! \ldots j_{m} !} p_{n}^{(\ell)}(\cos \theta)\left(\frac{-\sin \theta}{1 !}\right)^{j_{1}}\left(\frac{-\cos \theta}{2 !}\right)^{j_{2}} \ldots\left(\frac{\mathcal{D}^{m} \cos \theta}{m !}\right)^{j_{m}}
$$


where the sum is over all $m \geqq 1$ and $m$-tuples $\left(j_{1}, j_{2}, \ldots, j_{m}\right)$ of positive integers with $j_{1}+2 j_{2}+\cdots+m j_{m}=r$, while $\ell=j_{1}+j_{2}+\cdots+j_{m}$. From this, we see that $p_{n}^{(r)}$ arises only when $m=1, j_{1}=r$. Thus

$$
\mathcal{D}^{r}\left[p_{n}(\cos \theta)\right]=p_{n}^{(r)}(\cos \theta)(-\sin \theta)^{r}+\Sigma,
$$

where $\Sigma$ is a linear combination of $p_{n}^{(k)}(\cos \theta), 0 \leqq k \leqq r-1$, multiplied by powers of sin and cos. We then see that

$$
n^{-r}\left|\mathcal{D}^{r}\left[p_{n}(\cos \theta)\right]-p_{n}^{(r)}(\cos \theta)(-\sin \theta)^{r}\right| \leqq C_{2} \max _{0 \leqq k \leqq r-1} n^{-r}\left|p_{n}^{(k)}(\cos \theta)\right| .
$$

Applying (2.11), this last inequality, and using induction on $r$, we see that

$$
\sup _{x \in I_{1}}\left|p_{n}^{(r)}(x)\right| \leqq C n^{r}
$$

and hence

$$
\sup _{x \in I_{1}}\left|\mathcal{D}^{r}\left[p_{n}(\cos \theta)\right]-p_{n}^{(r)}(\cos \theta)(-\sin \theta)^{r}\right| \leqq C n^{r-1} .
$$

Finally (2.10) gives

$$
p_{n}^{(r)}(\cos \theta)(-\sin \theta)^{r}=2 \beta_{2 n} \operatorname{Re}\left[(-i n)^{r} e^{-i n \theta} \varphi_{2 n}^{*}\left(e^{i \theta}\right)\right]+o\left(n^{r}\right)
$$

and hence from (2.9),

$$
n^{-r} p_{n}^{(r)}(x)\left(1-x^{2}\right)^{r / 2}=\operatorname{Re}\left[i^{r} e^{-i n \theta} f\left(e^{-i \theta}\right)\right]+o(1) .
$$

Since (2.9) implies $f\left(e^{-i \theta}\right)=\overline{f\left(e^{i \theta}\right)}$ and since for any complex number $u$,

$$
\begin{gathered}
\operatorname{Re}\left[i^{r} u\right]= \begin{cases}(-1)^{r / 2} \operatorname{Re} \bar{u}, & \text { if } r \text { is even } \\
(-1)^{(r-1) / 2} \operatorname{Im} \bar{u}, & \text { if } r \text { is odd }\end{cases} \\
=(-1)^{[r / 2]} \mathcal{I}_{r}(\bar{u}),
\end{gathered}
$$

the result follows. 


\section{Proof of Theorems 4 and 6}

From (2.10),

$$
\begin{gathered}
{\left[\int_{0}^{\pi}\left|\mathcal{D}^{r}\left[p_{n}(\cos \theta)\right]-2 \beta_{2 n} \operatorname{Re}\left[(-i n)^{r} e^{-i n \theta} \varphi_{2 n}^{*}\left(e^{i \theta}\right)\right]\right|^{2} d \sigma(\theta)\right]^{1 / 2}} \\
\leqq 2\left|\beta_{2 n}\right| \sum_{j=1}^{r}\left(\begin{array}{c}
r \\
j
\end{array}\right) n^{r-j}\left[\int_{0}^{\pi}\left|\mathcal{D}^{j}\left[\varphi_{2 n}^{*}\left(e^{i \theta}\right)\right]\right|^{2} d \sigma(\theta)\right]^{1 / 2} \\
\leqq C n^{r-1}\left[\int_{0}^{\pi}\left|\mathcal{D} \varphi_{2 n}^{*}\left(e^{i \theta}\right)\right|^{2} d \sigma(\theta)\right]^{1 / 2}
\end{gathered}
$$

by repeated application of our Markov inequality (1.10). Next,

$$
\begin{gathered}
{\left[\int_{0}^{\pi}\left|\mathcal{D} \varphi_{2 n}^{*}\left(e^{i \theta}\right)\right|^{2} d \sigma(\theta)\right]^{1 / 2} \leqq\left[\int_{0}^{\pi}\left|\mathcal{D}\left(\varphi_{2 n}^{*}-\varphi_{[\sqrt{n}]}^{*}\right)\left(e^{i \theta}\right)\right|^{2} d \sigma(\theta)\right]^{1 / 2}} \\
+\left[\int_{0}^{\pi}\left|\mathcal{D} \varphi_{[\sqrt{n}]}^{*}\left(e^{i \theta}\right)\right|^{2} d \sigma(\theta)\right]^{1 / 2} \leqq C n\left[\int_{0}^{\pi}\left|\left(\varphi_{2 n}^{*}-\varphi_{[\sqrt{n}]}^{*}\right)\left(e^{i \theta}\right)^{2}\right| d \sigma(\theta)\right]^{1 / 2} \\
+C \sqrt{n}\left[\int_{0}^{\pi}\left|\varphi_{[\sqrt{n}]}^{*}\left(e^{i \theta}\right)\right|^{2} d \sigma(\theta)\right]^{1 / 2}=o(n),
\end{gathered}
$$

because of the classical Szegó asymptotics [11, p. 144] and (2.9)

$$
\lim _{m \rightarrow \infty} \int_{-\pi}^{\pi}\left|\varphi_{m}^{*}\left(e^{i \theta}\right)-D^{-1}\left(e^{i \theta}\right)\right|^{2} d \sigma(\theta)=0 .
$$

Recall here that $D(z)$ is the Szegó function, given by (1.3). Thus, from (3.1),

$$
\left[\int_{0}^{\pi}\left|n^{-r} \mathcal{D}^{r}\left[p_{n}(\cos \theta)\right]-2 \beta_{2 n} \operatorname{Re}\left[(-i)^{r} e^{-i n \theta} \varphi_{2 n}^{*}\left(e^{i \theta}\right)\right]\right|^{2} d \sigma(\theta)\right]^{1 / 2}=o(1)
$$

Using the just stated Szegó asymptotics (3.2), and the fact that $\beta_{2 n} \rightarrow 1 / \sqrt{2 \pi}$, we can restate this as

$$
\left[\int_{0}^{\pi}\left|n^{-r} \mathcal{D}^{r}\left[p_{n}(\cos \theta)\right]-\sqrt{\frac{2}{\pi}} \operatorname{Re}\left[(-i)^{r} e^{-i n \theta} D^{-1}\left(e^{i \theta}\right)\right]\right|^{2} d \sigma(\theta)\right]^{1 / 2}=o(1)
$$


The first part (1.11) of the theorem now follows. Next, let $I$ be a compact subinterval of $(-1,1)$. For each $r$, and all $n \geqq 1$,

$$
\int_{0}^{\pi}\left|n^{-r} \mathcal{D}^{r}\left[p_{n}(\cos \theta)\right]\right|^{2} d \sigma(\theta) \leqq C .
$$

This implies that for each $r$,

$$
\sup _{n \geqq 1} \int_{\tilde{I}}\left|n^{-r} p_{n}^{(r)}(\cos \theta)\right|^{2} d \sigma(\theta) \leqq C .
$$

This follows by an easy induction on $r$, using the identity (2.12), and the fact that $\sin \theta=\sqrt{1-x^{2}}$ is bounded below in $I$. Next, as at (2.13),

$$
\begin{gathered}
{\left[\int_{\tilde{I}}\left|n^{-r}\right| \mathcal{D}^{r}\left[p_{n}(\cos \theta)\right]-p_{n}^{(r)}(\cos \theta)(-\sin \theta)^{r}||^{2} d \sigma(\theta)\right]^{1 / 2}} \\
\leqq C \sum_{\ell=0}^{r-1}\left[\int_{\tilde{I}}\left|n^{-r} p_{n}^{(\ell)}(\cos \theta)\right|^{2} d \sigma(\theta)\right]^{1 / 2}=O\left(n^{-1}\right),
\end{gathered}
$$

by (3.5). This and (3.3) give

$$
\int_{\tilde{I}}\left|n^{-r} p_{n}^{(r)}(\cos \theta)(-\sin \theta)^{r}-\sqrt{\frac{2}{\pi}} \operatorname{Re}\left[(-i)^{r} e^{-i n \theta} D^{-1}\left(e^{i \theta}\right)\right]\right|^{2} d \sigma(\theta)=o(1) .
$$

Transferring this to the interval $I$ and taking account of whether $r$ is even or odd, gives (1.12).

Proof of Corollary 5. This is the case $r=1$ of (1.11), after a substitution.

Proof of Theorem 6. This is the same as that of Theorem 4, with obvious modifications.

\section{References}

[1] V. M. Badkov, Asymptotic and extremal properties of orthogonal polynomials in the presence of singularities in the weight, Proc. Steklov. Math. Inst., 198 (1994), $37-72$.

[2] V. M. Badkov, Pointwise estimates from below of the moduli of the derivatives of orthogonal polynomials on the circle with a weight having singularities, Mathematics USSR Sbornik, 186 (1995), 771-781.

[3] P. Borwein and T. Erdélyi, Polynomials and Polynomial Inequalities, Springer (New York, 1995). 
[4] G. Freud, Orthogonal Polynomials, Akadémiai Kiadó (Budapest, 1971).

[5] I. S. Gradshteyn and I. M. Ryzhik, Tables of Integrals, Series and Products, Academic Press (1980).

[6] D. S. Lubinsky, Asymptotics of derivatives of orthogonal polynomials on the unit circle, J. Approx. Theory., 145 (2007), 122-127.

[7] G. Mastroianni and V. Totik, Weighted polynomial inequalities with doubling and $A_{\infty}$ weights, Constr. Approx., 16 (2000), 37-71.

[8] A. Máté, P. Nevai and V. Totik, Strong and weak convergence of orthogonal polynomials, Amer. J. Math., 109 (1987), 239-282.

[9] K. T-R. McLaughlin and P. D. Miller, The D-steepest descent method and the asymptotic behavior of polynomials orthogonal on the unit circle with fixed and exponentially varying nonanalytic weights, manuscript.

[10] P. Nevai, An asymptotic formula for the derivatives of orthogonal polynomials, SIAM J. Math. Anal., 10 (1979), 472-477.

[11] B. Simon, Orthogonal Polynomials on the Unit Circle, Parts 1 and 2, American Mathematical Society (Providence, 2005).

[12] G. Szegô, Orthogonal Polynomials, 4th edn., American Math. Soc. Colloquium Publns., Vol. 23 (Providence, 1975).

[13] P. Vértesi, Uniform asymptotics of derivatives of orthogonal polynomials based on generalized Jacobi weights, Acta Math. Hungar., 85 (1999), 97-130. 